\title{
Article
}

\section{An Innovated Integrated Model Using Singular Spectrum Analysis and Support Vector Regression Optimized by Intelligent Algorithm for Rainfall Forecasting}

\author{
Weide Li ${ }^{*}$, Juan Zhang \\ School of Mathematics and Statistics, Lanzhou University, Lanzhou 730000, Gansu, China; weideli@lzu.edu.cn; \\ juanzhang15@lzu.edu.cn
}

\section{ABSTRACT}

Rainfall forecasting is becoming more and more significant and precipitation anomalies would lead to droughts and floods disasters. However, because of the complexity and non-stationary of rainfall data, it is difficult to forecast. In this paper, a novel hybrid model to forecast rainfall is developed by incorporating singular spectrum analysis (SSA) and dragonfly algorithm (DA) into support vector regression (SVR) method. Firstly, SSA is used for extracting the trend components of the hydrological data. Then, SVR is utilized to deal with the volatility and irregularity of the precipitation series. Finally, the parameter of SVR is optimized by DA. The proposed SSA-DA-SVR method is used to forecast the monthly precipitation for Songbai, Panshui, Lanma and Jiulongchi stations. To validate the efficiency of the method, four compared models, DA-SVR, SSA-GWO-SVR, SSA-PSO-SVR and SSA-CS-SVR are established. The result shows that the proposed method has the best performance among all five models, and its prediction has high precision and accuracy.

Keyword: Prediction; Precipitation; Singular Spectrum Analysis; Support Vector Regression; Intelligent Algorithm

ARTICLE INFO

Received: Apr 8, 2019

Accepted: Apr 29, 2019

Available online: May 15, 2019

*CORRESPONDING AUTHOR

Weide Li, School of Mathematics and

Statistics, Lanzhou University,

Lanzhou 730000, Gansu, China;

weideli@1zu.edu.cn;

\section{CITATION}

Weide Li, Juan Zhang. An Innovated

Integrated Model Using Singular

Spectrum Analysis and Support Vector

Regression Optimized by Intelligent

Algorithm for Rainfall Forecasting.

Journal of Autonomous Intelligence 2019

2(1): 46-55. doi: 10.32629/jai.v2i1.37

\section{COPYRIGHT}

Copyright (C) 2019 by author(s) and

Frontier Scientific Publishing. This

work is licensed under the Creative

Commons

Attribution-NonCommercial 4.0

International License (CC BY-NC4.0).

https://creativecommons.org/licenses/

by-nc/4.0/

\section{Introduction}

The precipitation will greatly affect people's life and production. If there is too much rainfall, it will lead to flash flooding and natural disasters, even though will cause severe economic losses and inconveniences to human life. As well known, the flooding is a life-threatening phenomenon ${ }^{[1-2]}$. If there is too little precipitation, it will exacerbate the conflict between domestic water use and irrigation water use, even lead to land salinization or desertification. Rainfall forecasting is a significant task for water resource management and the accuracy of the prediction is very important which affects the management of flood and drought. Meanwhile, rainfall forecasting is a difficult subject in hydrology because the involved physical processes is complex and the involved data, such as temperature, wind speed and it's direction are non-stationary in space and time.

In recently years, there are many methods used in hydrologic forecasting. Besides lots of mechanism models, data driving models are popular in recently years. They can be divided into two classification: probability statistics method and time series analysis method. The probability statistics method mainly includes: gray model (GM [1,1]), exponential and Markov model etc. These methods can only be used on the exponential growth of rainfall forecasting. The time series analysis method mainly includes auto regression (AR), moving average auto-regression 
(MA) and auto-regression integrating moving average (ARIMA), etc ${ }^{[3-5]}$. Their prediction value tends to average, so it is inaccurate to predict extreme values. Because the weather condition has variability, diversity and complexity, rainfall is uncertain and random which is a nonlinear time series. Therefore, it is difficult to forecast the rainfall by the traditional methods. In the past few years, the computational intelligence approaches known as machine learning become more and more popular, and they have been used in rainfall forecasting in hydrological area. These methods include: artificial neural network ${ }^{[6-9]}$, support vector regression ${ }^{[10-12]}$, extreme learning machine, ensemble empirical mode decomposition ${ }^{[13]}$, singular spectrum analysis $^{[14]}$, and all kinds of heuristic optimization algorithm $^{[15-17]}$.

Recently, using combined methods to predict time series is developing fast in different fields ${ }^{[18-20]}$. The idea to hybrid data preprocessing method, forecasting model and optimization method to predict rainfall is attractive. In this paper, based on singular spectrum analysis (SSA), dragonfly algorithm (DA) and support vector regression (SVR), a hybrid model SSA-DA-SVR is proposed to deal with the hydrologic problem.

The remaining of the paper is organized as follows: The related methods are introduced in section 2 and the hybrid model is put forward and the test indicators are described. The case analysis is conducted on four different stations and the results of proposed model are compared with DA-SVR, SSA-GWO-SVR, SSA-PSO-SVR, and SSA-CS-SVR models in section 3. Finally, the conclusion is given in section 4 .

\section{Related methods}

\subsection{Singular spectrum analysis (SSA)}

SSA is a method studying nonlinear time sequences, which used to avoid the effect of discontinuous signals. It can be combined with neural networks for time series forecasting. The applications of SSA include smoothing, trend extraction and filtering. Based on the observed time series, construct the trajectory matrix, and identify and extract the periodic, qua-periodic and oscillatory components from the original data ${ }^{[21]}$. Standard SSA performs four steps, which include embedding, singular value decomposition (SVD), grouping and diagonal averaging. The first two steps called the time series decomposition and the after two steps know as the reconstruction. The algorithm of SSA is simply showed as follows:

Stage 1: Decomposition stage

First step : Embedding. It can be regarded as a mapping which converts the one-dimensional original series $Y=\left[y_{1}, y_{2}, \ldots, y_{N}\right]$ into the multidimensional vector $\quad\left(X_{1}, X_{2}, \ldots X_{n}\right), \quad$ where $X_{i}=\left(y_{i}, y_{i+1}, \cdots y_{i+\mathrm{m}-1}\right)^{T} \in \mathfrak{R}^{m}, i=1,2, \cdots, n \quad$ and $n=N-m+1$. The vectors, called L-lagged vectors, are grouped into the trajectory matrix

$$
X=\left[\begin{array}{cccc}
y_{1} & y_{2} & \cdots & y_{K} \\
y_{2} & y_{3} & \cdots & y_{K+1} \\
\cdots & \cdots & \ddots & \cdots \\
y_{L} & y_{L+1} & \cdots & y_{N}
\end{array}\right]
$$

where $m(2 \leq m \leq N)$ is the selected embedded dimension, and $\mathrm{m}$ should not be greater than one second of the series length ${ }^{[22]}$.

Second step : SVD. It can be defined as follows:

$$
X_{n \times m}=U_{n \times m} \Sigma_{m \times m} V_{m \times m}^{T}
$$

where $X_{n \times m}$ is the trajectory matrix, $U_{n \times m}$ is the left singular matrix, $V_{m \times m}$ is the right singular matrix, $\Sigma_{m \times m}$ is the diagonal matrix. Its diagonal element is the singular value. Let $A=X^{T} X$, the eigenvalues of $A$ is $\lambda_{1}, \lambda_{2}, \cdots, \lambda_{m}\left(\lambda_{1} \geq \lambda_{2} \geq \cdots \geq \lambda_{m}\right) \quad$ by $\left(X^{T} X\right) v_{i}=\lambda_{i} v_{i}$. Where $v_{i}$ is the eigenvector of $\lambda_{i}$, and $v_{i}$ can construct the right singular matrix $V_{m \times m}$ in Eq (2). The left singular matrix $U_{n \times m}$ in Eq (2) can be calculated by Eq (3) and Eq (4):

$$
\begin{aligned}
\alpha_{i} & =\sqrt{\lambda_{i}} \\
u_{i} & =\frac{1}{\alpha_{i}} X V_{i}
\end{aligned}
$$

where $\alpha_{i}$ is the singular value, $u_{i}$ the left singular vector.

The singular value $\alpha_{i}$ is similar to the eigenvalues, and they are also arranged in decreasing order in the matrix $\Sigma_{m \times m}$, and the reduction of $\alpha_{i}$ is particularly fast. In many cases, the sum of the singular values of the 
first $10 \%$ or even $1 \%$ accounts for more than $99 \%$ of the sum of all the singular values. In other words, we can also use the former $r$ large singular values to approximate the matrix:

$$
X_{m \times n} \approx U_{m \times r} \Sigma_{r \times r} V_{r \times n}^{T},
$$

where $r$ is a number that is much smaller than $\mathrm{m}, \mathrm{n}$, and the result of the multiplication of the three matrices on the right will be a matrix close to $X$. When $r$ is closer to n, the result of multiplication is closer to $X$.

Stage 2 : Reconstruction stage

First step: Suppose there are N singular values $\alpha_{1}, \alpha_{2}, \cdots \alpha_{N}$. We define the contribution rates of the $i$-th singularity as follows:

$$
m_{i}=\frac{\sigma_{i}}{\sum_{i=1}^{m} \sigma_{i}}
$$

We choose front (from large to small row) $r$ singular values so as to their contribution rate being greater than a certain threshold (for example, 80\%).

Second step : Diagonal averaging.

Assuming that the reconstructed matrix after SVD decomposition as follows:

$$
X=\left(x_{i j}\right)_{i, j=1}^{n, m}=\left[\begin{array}{ccccc}
x_{1,1} & x_{1,2} & x_{1,3} & \cdots & x_{1, m} \\
x_{2,1} & x_{2,2} & x_{2,3} & \cdots & x_{2, m} \\
\vdots & \vdots & \vdots & \cdots & \vdots \\
x_{n, 1} & x_{n, 2} & x_{n, 3} & \cdots & x_{n, n+m-1}
\end{array}\right]
$$

then the reconstructed series $Y=\left[y_{1}, y_{2}, \cdots, y_{N}\right]$ is computed using diagonal averaging method that is defined as follows:

$$
\begin{gathered}
y_{p}=\left(\sum_{j=1}^{m} x_{i, j}\right) / \beta_{p}, i=p-j+1,0<i<n+1, \\
\beta_{p}=\left\{\begin{array}{cc}
p & p<m, \\
T-p+1 & p>T-m+1, \\
m & \text { otherwise }
\end{array}\right.
\end{gathered}
$$

where $\quad x_{i j}(i=1, \cdots, n, j=1, \cdots, i+m-1) \quad$ is the element of reconstructed trajectory matrix $X$.

\subsection{Dragonfly algorithm (DA)}

In 2015, Mirjalili proposed a novel swarm intelligent optimization algorithm called dragonfly algorithm (DA) ${ }^{[23]}$. This algorithm is mainly based on the static and dynamic swarm intelligence behaviors of dragonflies, such as the navigation of dragonflies, the search for food, the avoidance of enemy in mathematical modeling. Mirjalili proved that for a given problem, DA can effectively improve the initial random population, and can converge to the global optimal solution. Compared with other known optimization algorithms, DA has a competitive advantage. The algorithm is as follows:

1) Update the dragonfly position vector $S_{i}, A_{\mathrm{i}}, C_{i}, F_{i}$ and $E_{i}$. Here $S_{i}, A_{i}, C_{i}, F_{i}$ and $E_{i}$ respectively represent separation, alignment, cohesion, food source, and the position of enemy.

2) Compute the step vector:

$$
\Delta X_{t+1}=\left(s S_{i}+a A_{i}+c C_{i}+f F_{i}+e E_{i}\right)+\omega \Delta X_{t}
$$

3) Update position vector:

when we have a neighbor,

$$
X_{t+1}=X_{t}+\Delta X_{t+1}
$$

no neighbors,

$$
X_{t+1}=X_{t}+\operatorname{Levy}(d) \times X_{t},
$$

where $d$ is the dimension of the position vectors.

The Levy flight is calculated as following:

$$
\operatorname{Levy}(x)=0.01 \times \frac{r_{1} \times \delta}{\left|r_{2}\right|^{\frac{1}{\beta}}},
$$

where $r_{1}, r_{2}$ are two random numbers in $[0,1] . \quad \beta$ is a constant, and $\delta$ is calculated as follows:

$$
\delta=\left(\frac{\Gamma(1+\beta) \times \sin \left(\frac{\pi \beta}{2}\right)}{\Gamma\left(\frac{1+\beta}{2}\right) \times \beta \times 2^{\frac{\beta-1}{2}}}\right)^{\frac{1}{\beta}}
$$

where $\Gamma(x)=(x-1) !$

\subsection{Support vector regression(SVR)}

Recent developments in intelligent methods allow their utilization in complex system modeling. The support vector machine (SVM) proposed by Vapnik and his colleagues ${ }^{[24]}$ is a popular methodology that has been successfully utilized for pattern recognition, 
An Innovated Integrated Model Using Singular Spectrum Analysis and Support Vector Regression Optimized by Intelligent Algorithm for Rainfall Forecasting

classification and regression. SVR, a version of SVM for regression, was proposed by Vapnik, Steven Golowich and Alex Smola in $1997^{[25]}$. One primary characteristic of SVR is that rather than minimizing the observed training error, SVR attempts to minimize the generalized error bound to achieve generalized performance ${ }^{[26-27]}$. Figure 1 shows the basic principle of SVR.

\subsection{The proposed hybrid model}

In order to deal with the short-term forecasting problem of the rainfall data, an integrated model, SSA-DA-SVR, is proposed, based on SSA, DA, and SVR. SSA is used to process the monthly data, the

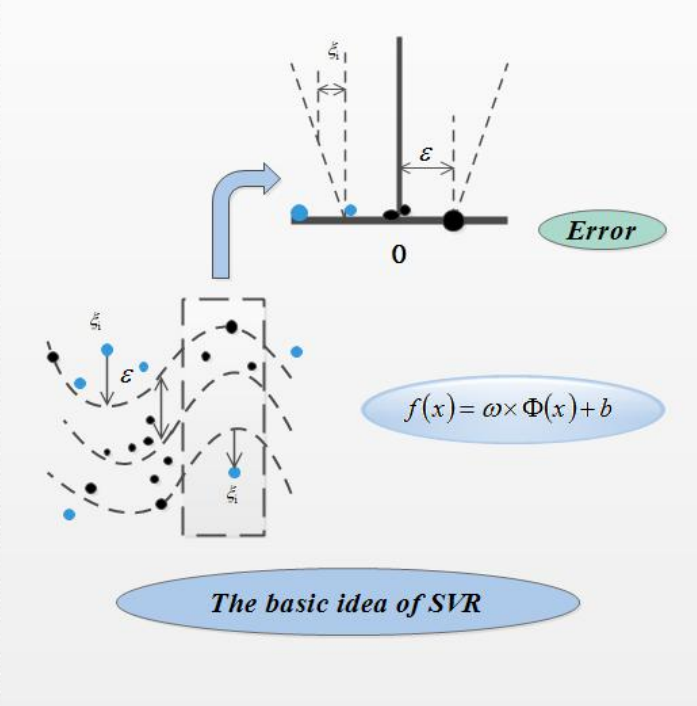

filtered time series by SSA are used for prediction by SVR, while DA is used to optimize the two parameters c and $g$ in the SVR model. The basic algorithm of the hybrid model is as follows:

Step 1: Rainfall data preprocessing.

Step 2: Decomposition and reconstruction. The reconstructed sequence of several components is obtained through the singular spectrum decomposition technique.

Step 3: The prediction of the reconstructed sequence. SVR algorithm coupled with dragonfly algorithm is used to train the model and predict the rainfall.

SVR uses structural risk minimization, which minimizes both empirical risk and the model complexity simultaneously, instead of the empirity risk used in methods such as artificial neural networks. When the structural risk is minimized, the model is able to optimize its date generalization. A model that generalizes well is able to correctly make a prediction when new examples are introduced

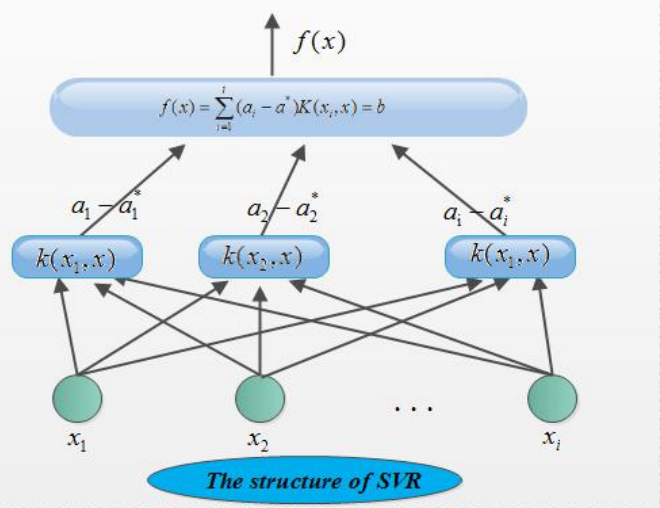

Figure 1. The basic idea and structure of SVR

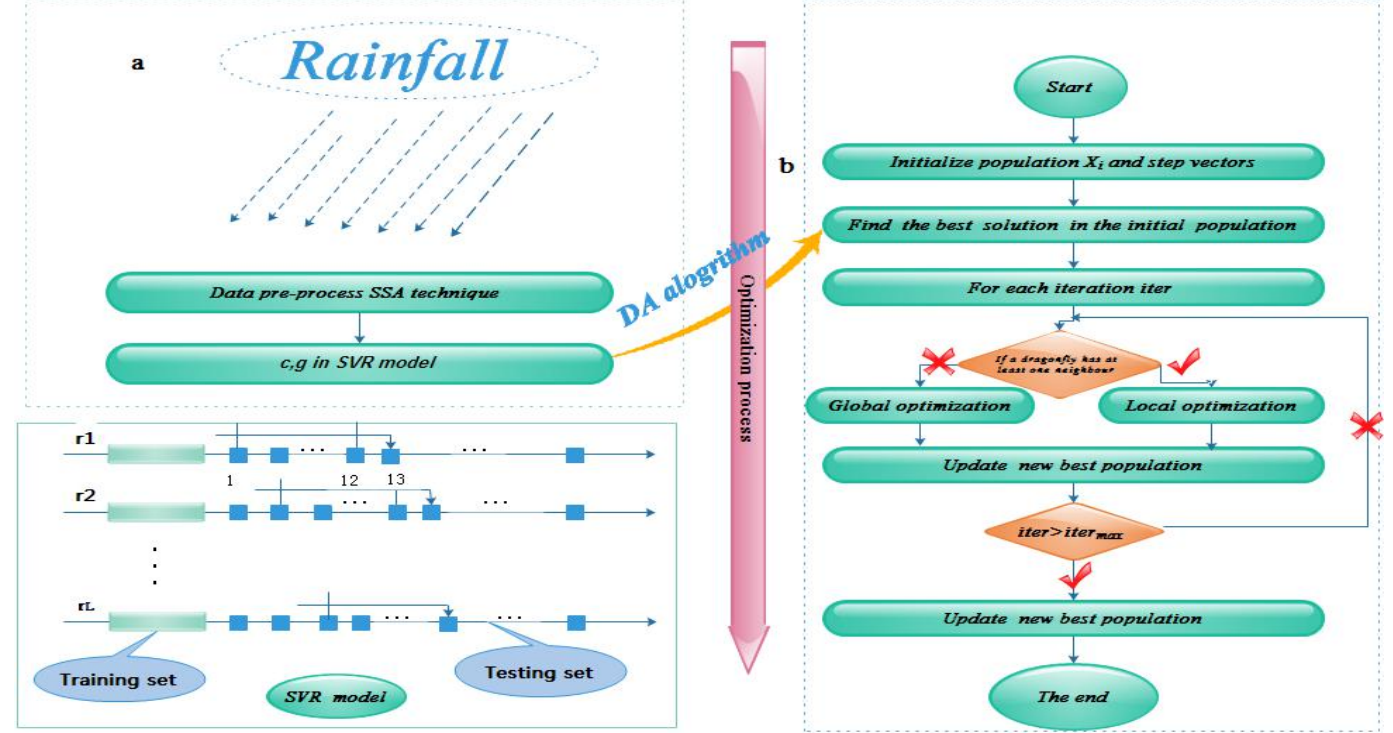

Figure 2. The basic structure of SSA-DA-SVR model 
An Innovated Integrated Model Using Singular Spectrum Analysis and Support Vector Regression Optimized by Intelligent Algorithm for Rainfall Forecasting

\subsection{Predictive performance assessment indicator}

In order to evaluate the proposed hybrid forecasting method, four evaluation indicators are used: mean absolute error (MAE), root mean square of error (RMSE), Nash-Sutcliffe coefficient of efficiency (NS) and correlation $\left(\mathrm{R}^{2}\right)$. The specific formula is as following.

$$
\begin{gathered}
M A E=\frac{1}{N} \sum_{i=1}^{N}\left|y_{i}-\hat{y_{i}}\right| \\
R M S E=\sqrt{\frac{1}{N} \sum_{i=1}^{N}\left(y_{i}-\hat{y}_{i}\right)^{2}} \\
N S=1-\frac{\sum_{i=1}^{N}\left(y_{i}-\hat{y}_{i}\right)^{2}}{\sum_{i=1}^{N}\left(y_{i}-\bar{y}\right)^{2}} \\
R^{2}=\frac{1}{N-1} \sum_{i=1}^{N}\left(\frac{X_{i}-\bar{X}}{S_{X}}\right)\left(\frac{Y_{i}-\bar{Y}}{S_{Y}}\right)
\end{gathered}
$$

where $y_{i}$ and $\hat{y}_{i}$ are the actual and forecasting values of time series respectively, and $\mathrm{N}$ is the number of prediction data points. $X_{i}, Y_{i}$ and $\bar{X}, \bar{Y}$ are two variables' observed values and means, respectively, and $S_{X}, S_{Y}$ are the variances of the two variables, respectively.

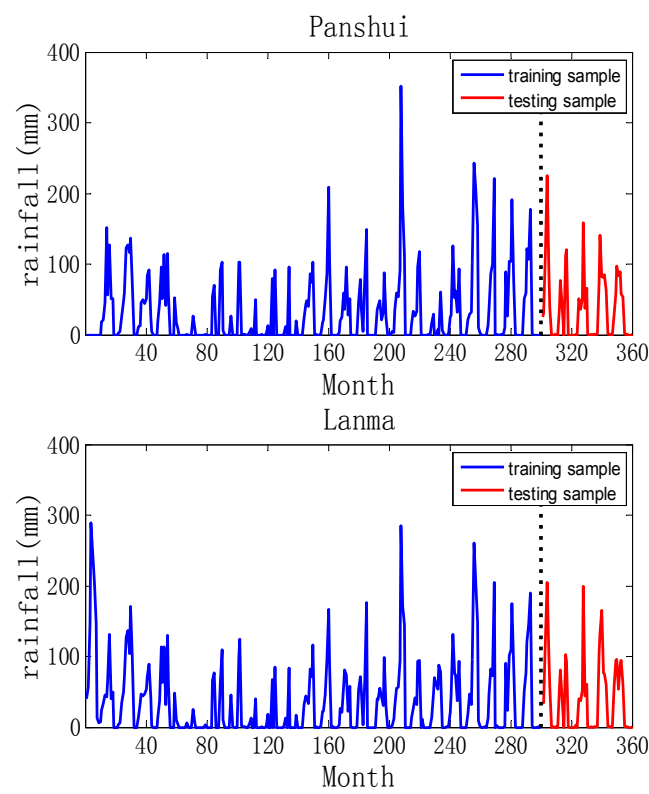

\section{Experimentation results}

To validate the effectiveness of the improved novel integrated model, two experiments are designed on monthly average rainfall data at four different rainfall stations.

\subsection{Study area and data sets}

The case study is conducted on four rainfall stations, Songbai, Panshui, Lanma, and Jiulongchiin in Shenglongjia region of Hubei province, China. The area of Shenglongjia region is $3225 \mathrm{~km}^{2}$ and its annual average temperature is $11.00^{\circ} \mathrm{C} \sim 12.2^{\circ} \mathrm{C}$. The region has abundant precipitation, with the annual average rainfall is $1170.2 \mathrm{~mm}$, and its water vapor is mainly supplied by southeast and southwest. About $80 \%$ the annual precipitation is from April to September, and in winter, with less precipitation. Figure 3 shows the monthly average precipitation data from April 1983 to March 2013 collecting from Songbai, Panshui, Lanma and Jiulongchi four stations. There are 360 samples on every station. And the 1st-300th are used as training samples to build forecasting models, and the 301st-360th are used as testing samples to validate the models. Considering the periodicity of the monthly average rainfall, the former 12 data as input data, namely, the 13th data point is predicted by the 1st-12th data points, the 14th data point is predicted by the $2 n d-13$ th data points, and so on.
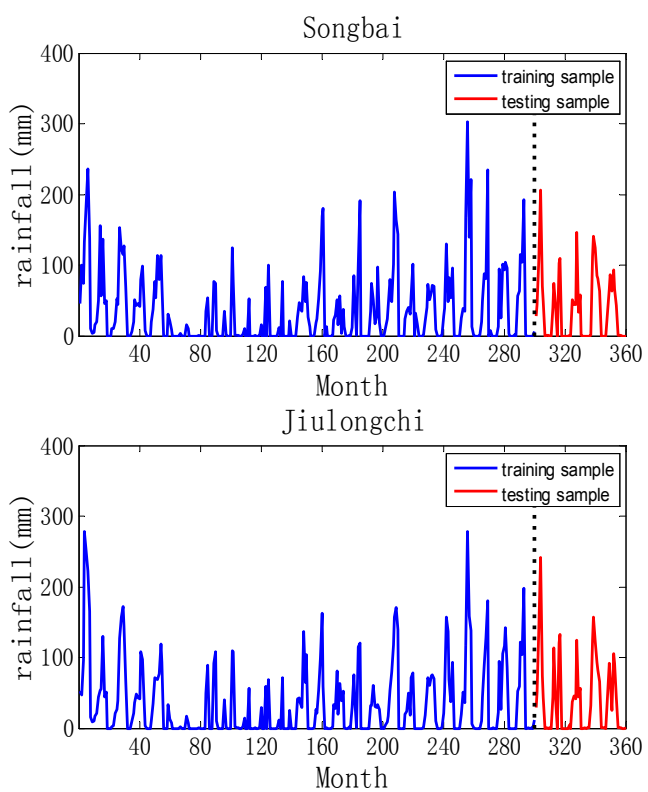

Figure 3. Original rainfall series from four stations 
An Innovated Integrated Model Using Singular Spectrum Analysis and Support Vector Regression Optimized by Intelligent Algorithm for Rainfall Forecasting

\subsection{Experimental setup}

\subsubsection{Experiment I : Results of data preprocessing}

In this experiment, in order to verify the role of SSA, we compared the performances of SSA-DA-SVR model with that of DA-SVR model. In the SSA process, the used window length is half the length of the entire time series, and the number of the principle components is determined by the contribution rate. In the case of Panshui, we use $\mathrm{L}=170, \mathrm{r}=26$. The other three stations use similar parameters $\mathrm{L}$, meanwhile, the values of $r$ are $30,28,33$ on Songbai, Jiulongchi and Lanma respectively.

Table 1 shows the results of SSA-DA-SVR and DA-SVR models in terms of four assessment criteria,
MAE, RMSE, NS and $\mathrm{R}^{2}$ on the four researched stations. The values in bold represent the smaller one of MAE and RMSE values and larger one of NS and $\mathrm{R}^{2}$ among two models at the four stations. The original rainfall sequences is preprocessed by SSA before forecasting by SSA-DA-SVR and the original rainfall series is used forecasting by DA-SVR in four stations. Figure 4(a) illustrated the original reconstructed rainfall series, and Figure 4(b) presented the residual series. From Figure 5, we can see that the curve of the proposed integrated model predicted is closer to the curve of the original data compared with that of DA-SVR model predicted in four stations. Above results show that SSA technique effectively improves the prediction accuracy of the combined model .

\begin{tabular}{llllll}
\hline Station & model & MAE & RMSE & NS & $\mathbf{R}^{2}$ \\
\hline \multirow{2}{*}{ Panshui } & SSA-DA-SVR & 5.6120 & 7.4430 & 0.9751 & 0.9782 \\
& DA-SVR & 23.8732 & 30.4194 & 0.3430 & 0.5383 \\
Songbai & SSA-DA-SVR & 4.3965 & 5.0821 & 0.9896 & 0.9929 \\
& DA-SVR & 23.5351 & 32.0921 & 0.3152 & 0.4828 \\
Jiulongchi & SSA-DA-SVR & 6.1809 & 7.6839 & 0.9730 & 0.9776 \\
& DA-SVR & 23.9479 & 33.021 & 0.3324 & 0.5351 \\
\multirow{2}{*}{ Lanma } & SSA-DA-SVR & 4.5307 & 5.4179 & 0.9878 & 0.9898 \\
& DA-SVR & 25.7736 & 34.7035 & 0.3458 & 0.5123 \\
\hline
\end{tabular}

Table 1. Forecasting results of SSA-DA-SVR and DA-SVR models for four stations

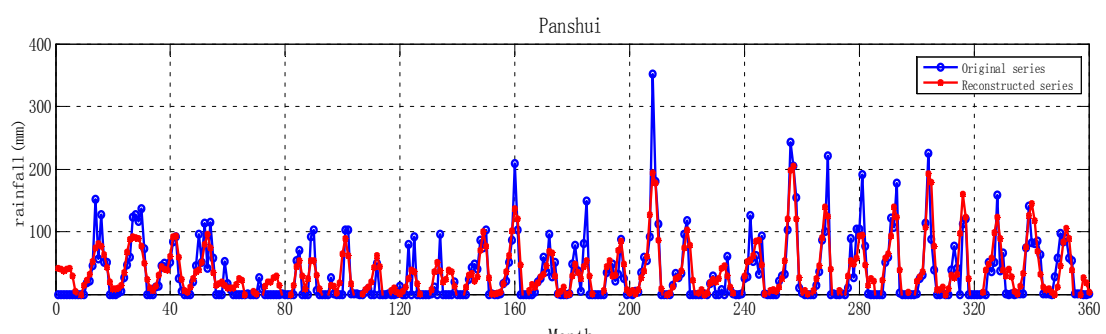

Month

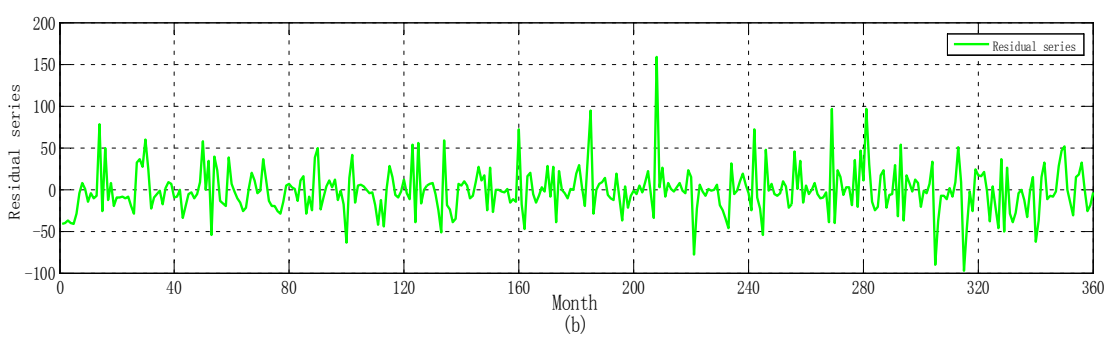

Figure 4. Rainfall series ((a) original, reconstructed series and (b) residual series for $\mathrm{L}=170$ and $\mathrm{r}=26$ in Panshui stations) 

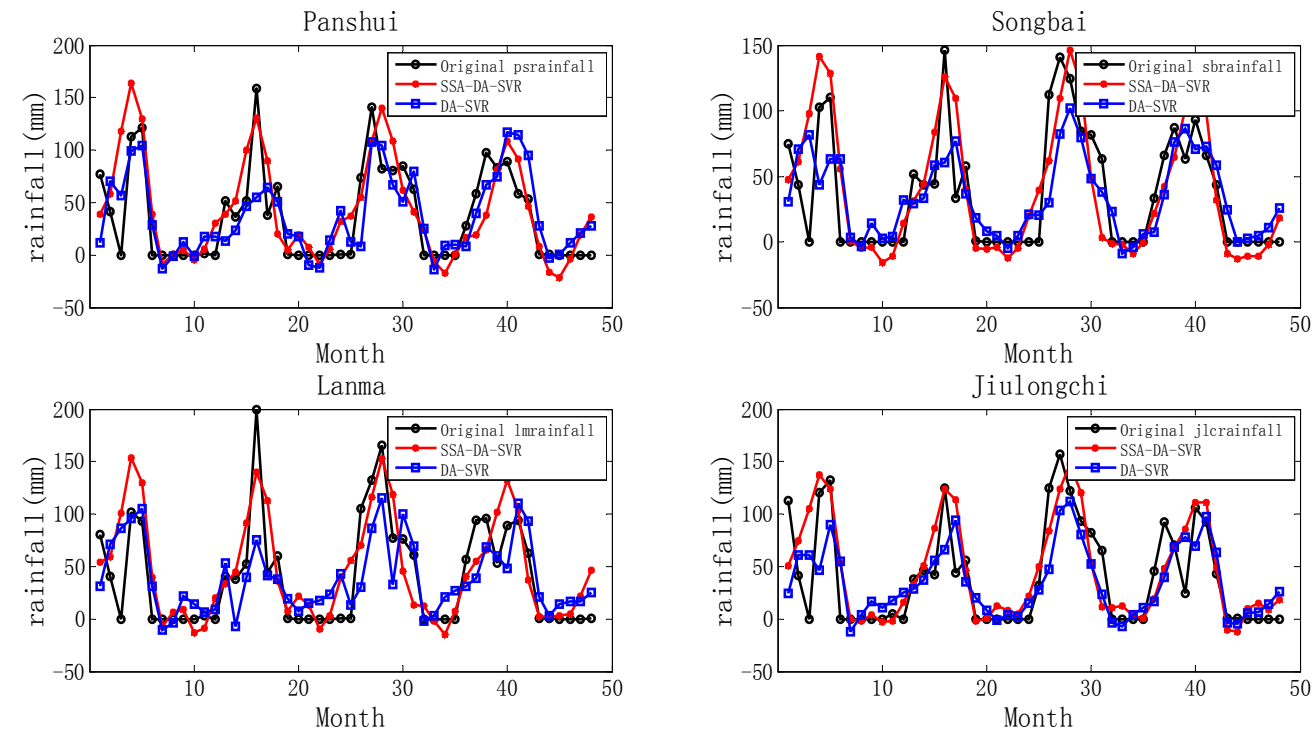

Figure 5. The results of SSA-DA-SVR and DA-SVR models in four stations

\subsubsection{Experimental II: Forecasting comparison results}

In this part, the proposed model SSA-DA-SVR is compared with other three forecasting models, namely, SSA-GWO-SVR, SSA-PSO-SVR, and SSA-CS-SVR. The results are demonstrated in Table 2 and Figure 6.

Table 2 illustrates the values of the four evaluation criteria on the forecasts offered by SSA-DA-SVR and that offered by the three compared models among the four stations. It is clearly seen that SSA-DA-SVR model can provide high forecasting accuracy. For example, at Jiulongchi station, the value of $\mathrm{NS}$ and $\mathrm{R}^{2}$ of SSA-DA-SVR models are $0.9730,0.9776$ respectively, while the value of NS and $\mathrm{R}^{2}$ of other three models are all smaller than that of the proposed model. The value of MAE of SSA-DA-SVR is 6.1809, while the value of MAE of SSA-GWO-SVR, SSA-PSO-SVR, SSA-CS-SVR models are 8.5026, 12.3819, 9.9583 respectively. The results indicate that the DA algorithm effectively improves the prediction accuracy of the proposed integrated model. Figure 6 illustrates the predicted values of the four models and actual values on four stations. It can be seen that the predicted cure of SSA-DA-SVR is the best one to approximate the actual one in all four compared models.

\begin{tabular}{llllll}
\hline Station & model & MAE & RMSE & NS & $\mathbf{R}^{\mathbf{2}}$ \\
\hline Panshui & SSA-DA-SVR & 5.6120 & 7.4430 & 0.9751 & 0.9782 \\
& SSA-GWO-SVR & 9.0853 & 10.8052 & 0.9483 & 0.9503 \\
& SSA-PSO-SVR & 11.2609 & 14.2797 & 0.9322 & 0.9375 \\
Songbai & SSA-CS-SVR & 11.2759 & 14.2896 & 0.9070 & 0.9187 \\
& SSA-DA-SVR & 4.3965 & 5.0821 & 0.9896 & 0.9929 \\
& SSA-GWO-SVR & 8.4167 & 10.5156 & 0.9429 & 0.9514 \\
& SSA-PSO-SVR & 10.7035 & 13.1829 & 0.9152 & 0.9235 \\
\hline \multirow{2}{*}{ jiulongchi } & SSA-CS-SVR & 10.3600 & 13.6768 & 0.9063 & 0.9180 \\
& SSA-DA-SVR & 6.1809 & 7.6839 & 0.9730 & 0.9776 \\
& SSA-GWO-SVR & 8.5026 & 10.4693 & 0.9556 & 0.9575 \\
& SSA-PSO-SVR & 12.3819 & 16.0805 & 0.9030 & 0.9098 \\
\hline Lanma & SSA-CS-SVR & 9.9583 & 12.7174 & 0.9362 & 0.9376 \\
\hline & SSA-DA-SVR & 4.5307 & 5.4179 & 0.9878 & 0.9898 \\
& SSA-GWO-SVR & 9.4084 & 11.6235 & 0.9433 & 0.9446 \\
\hline
\end{tabular}


An Innovated Integrated Model Using Singular Spectrum Analysis and Support Vector Regression Optimized by Intelligent Algorithm for Rainfall Forecasting

\begin{tabular}{lllll}
\hline SSA-PSO-SVR & 9.5149 & 11.8446 & 0.9405 & 0.9324 \\
SSA-CS-SVR & 10.3539 & 12.7402 & 0.9345 & 0.9360 \\
\hline
\end{tabular}

Table 2. Evaluation criteria of four forecasting models at four stations
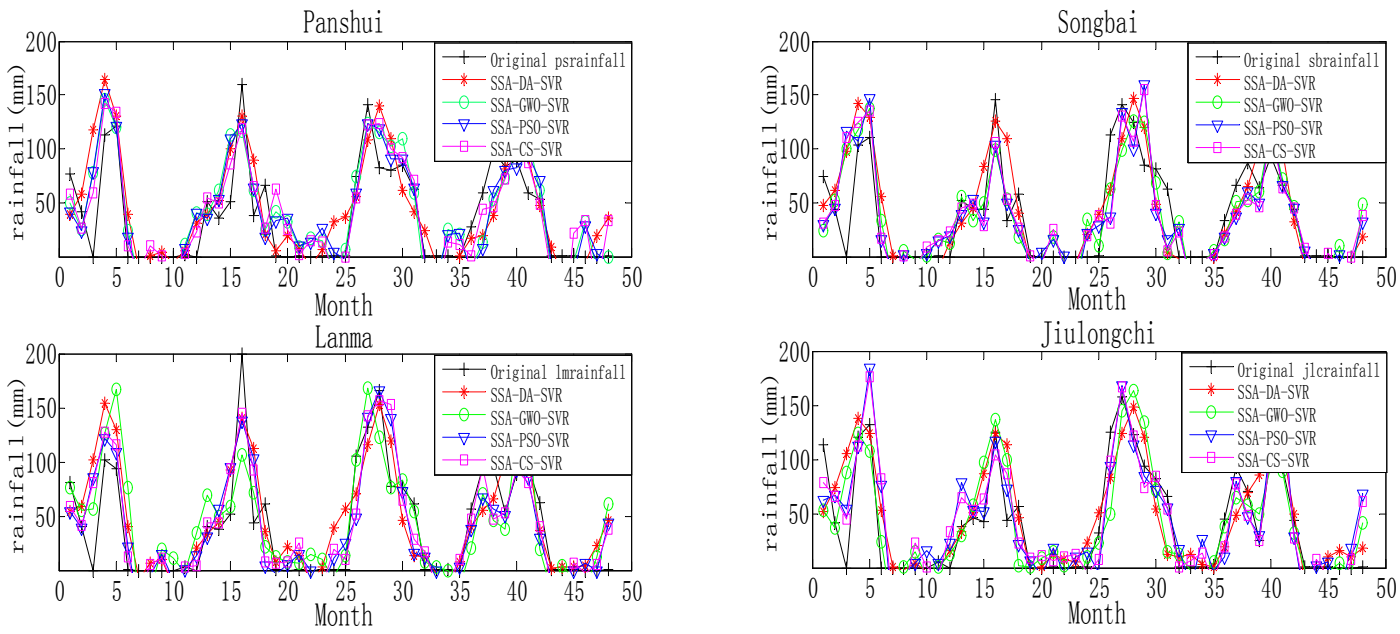

Figure 6. Comprehensive assessment of forecasting models at four stations. Forecasting results of the four predicting models and actual values for the four stations are illustrated

\section{Conclusions}

Rainfall projections play a crucial role in our life and industry. Accurate precipitation forecasting is instrumental in floods prevention and drought management. Therefore, in this study, we proposed SSA-DA-SVR model for predicting the average month precipitation in Panshui, Songbai, Jiulongchi and Lanma stations. Compared with DA-SVR, SSA-GWO-SVR, SSA-PSO-SVR, SSA-CS-SVR models, the proposed hybrid model can effectively improve the prediction accuracy for month average precipitation. Thus, the model can be used on rainfall forecasting in the future. In addition, as a prediction model, it can also be applied in wind speed and power load forecasting ${ }^{[28]}$. Although the proposed model has obvious advantages in month average precipitation forecasting, there are still many techniques can be used to improve the accuracy of prediction. For example, in order to improve predictive performance, we can use $\mathrm{VMD}^{[29]}$, ICEEMD[30] to replace SSA in procedure of processing.

\begin{tabular}{llll}
\multicolumn{2}{l}{ Nomenclature } & & \\
AR & auto regression & & \\
ARIMA & auto-regression & integrating & moving \\
CS & average & &
\end{tabular}

cuckoo search algorithm

DA dragonfly algorithm

GM grey models

GWO grey wolf optimization algorithm

ICEEMD improved complete ensemble empirical mode decomposition

MA moving average auto-regression

MAE mean absolute error

NS nash-sutcliffe coefficient of efficiency

PSO particle swarm optimization

RMSE root mean square error

SSA singular spectrum analysis

SVD singular value decomposition

SVM support vector machine

SVR support vector regression

VMD variational mode decomposition

\section{Acknowledgements}

This research is supported by the National Natural Sciences Foundation of China(No. 40571016) and the National Key Research and Development Program of China (Grant No. 2018YFC0406606).

\section{Conflicts of Interest}

The authors declare no conflict of interest. 


\section{References}

1. J. Wu. A Novel Artificial Neural Network Ensemble Model based on K--Nearest Neighbor Nonparametric Estimation of Regression Function and Its Application for Rainfall Forecasting[C]// International Joint Conference on Computation Sciences and Optimization. IEEE, 2009, 2, 44-48.

2. CM. Tseng, CD. Jan, JS. Wang and CM. Wang. Application of artificial neural network in typhoon surge forecasting. Ocean Engineering. 2007, 34, 1757-1768.

3. P. Narayanna, A. Basistha, S. Sarkar, S. Kamna. Trend analysis and ARIMA modelling of pre-monsoon rainfall data for western India. Comptes Rendus Geoscience. 2013, 345, 22-27.

4. S. Chattopadhyay, G. Chattopadhyay. Univariate modelling of summer-monsoon rainfall time series: Comparison between ARIMA and ARNN. Comptes Rendus Geoscience. 2010, 342, 100-107.

5. S. Afshin, H. Fahmi, A. Alizadeh, H. Sedghi, F. Kaveh. Long term rainfall forecasting by integrated artificial neural network-fuzzy logic-wavelet model in Karoon basin. Scientific Research and Essays. 2011, 6, 1200-1208.

6. C. Jareanpon, W. Pensuwon, RJ. Frank and N. Davey. An Adaptive RBF network optimised using a genetic algorithm applied to rainfall forecasting. International Symposion on Communications and Information Technologies. IEEE, 2004, 2, 1005-1010.

7. M. Anwer, K. Khan, S. Banik, FH. Chanchary. Forecasting Bangladesh Monsoon Rainfall Neural Network and Genetic Algorithm Approaches. World Journal of Microbology \& Biotechnology. 2009, 2, $1-18$.

8. M. Nasseri, K. Asghari, MJ. Abedini. Optimized scenario for rainfall forecasting using genetic algorithm coupled with artificial neural network. Expert Systems with Applications. 2008, 35, 1415-1421.

9. Riccardo Taormina, Kwok-Wing Chau. Data-driven input variable selection for rainfall-runoff modeling using binary-coded particle swarm optimization and Extreme Learning Machines. Journal of Hydrology. 2015, 529, 1617-1632.

10. CC. Young, WC. Liu, MC. Wu. A physically based and machine learning hybrid approach for accurate rainfall-runoff modeling during extreme typhoon events. Applied Soft Computing. 2017, 53, 205-216

11. SM. Hosseini, N. Mahjouri. Integrating Support Vector Regression and a geomorphologic Artificial Neural Network for daily rainfall-runoff modeling. Applied Soft Computing. 2016, 38, 329-345.

12. MC. Wu, GF. Lin, HY. Lin. Improving the forecasts of extreme streamflow by support vector regression with the data extracted by self-organizing map. Hydrological Processes. 2012,
28, 386-397.

13. WC. Wang, KW. Chau, L. Qiu, YB. Chen. Improving forecasting accuracy of medium and long-term runoff using artificial neural network based on EEMD decomposition. Environmental Research. 2015, 139, 46-54.

14. KW. Chau, CL. Wu. A hybrid model coupled with singular spectrum analysis for daily rainfall prediction. Journal Hydroinformatics. 2010, 12, 458-473.

15. KS. Kasiviswanathan, R. Cibin, KP. Sudheer, I. Chaubey. Constructing prediction interval for artificial neural network rainfall runoff models based on ensemble simulations. Journal of Hydrology. 2013, 499, 275-288.

16. Nasseri M, Asghari K, Abedini M. Optimized scenario for rainfall forecasting model using genetic algorithm coupled with artificial neural network. Expert Systems with Applications. 2008, 35, 1415-1421.

17. J. Wu, J. Long, M. Liu. Evolving RBF neural networks for rainfall prediction using hybrid particle swarm optimization and genetic algorithm. Neurocomputing. 2015, 148, 136-142.

18. Mingfei Niu, Shaolong Sun, Jing Wu, Lean, Jianzhou Wang. An innovative integrated model using the singular spectrum analysis and nonlinear multi-layer perception network optimized by hybrid intelligent algorithm for short-term load forecasting. Applied Mathematical Modelling. 2016, 40, 4079-4093.

19. Y. Wang, J. Wang, X. Wei. A hybrid wind speed forecasting model based on phase space reconstruction theory and Markov model : A case study of wind farms in northwest China. Energy. 2013, 91, 556-572.

20. Y. Xu, W. Yang, J. Wang. Air quality early-warning system for cities in China. Atmospheric Environment. 2017, 148, 239-257.

21. M. Abdollahzade, et al. A new hybrid enhanced local linear neruo-fuzzy model based on the optimized singular spectrum analysis nonlinear and chaotic time series forecasting. Information Sciences. 2015, 295, 107-125.

22. N. Golyandina, V. Nekrutkin, A. Zhigljavsky, Analysis of Time Series Structure: SSA and Related Techniques, CRC Press, 2001.

23. S. Mirjalili. Dragonfly algorithm: a new meta-heuristic optimization technique for solving single-objective, discrete, and multi-objective problems. Neural Computing \& Applications. 2016, 27, 1053-1073.

24. V. Vapnik, S. Golowich, A. Smola . Support vector method for function approximation, regression estimation, and signal processing. In:Mozer MC, Jordan MI, Petsche T, editors. Neural information processing systems. 1997, 9, 281-287.

25. D. Basak, DC. Patranabis, S. Pal . Support vector regression. Neural Information Processing- Letters 
An Innovated Integrated Model Using Singular Spectrum Analysis and Support Vector Regression Optimized by Intelligent Algorithm for Rainfall Forecasting

Reviews. 2007, 11, 203-224.

26. WC. Hong, YC. Dong, WY. Zhang, LY. Chen, BK. Panigrahi. Cyclic electric load forecasting by seasonal SVR with chaotic genetic algorithm . Electr Power Energy Syst. 2013, 44, 604-614.

27. AJ. Smola, B. Scholkopf. A tutorial on support vector regression. Statist. Comput. 2004, 14, 199-222.

28. WD. Li, DM. Kong, JR. Wu. A novel hybrid model based on extreme learning machine, k-nearest neighbor regression and wavelet denoising applied to short-term electric load forecasting. Energies. 2017; 10, 694; doi: 10.3390/en10050694.

29. K. Dragomiretskiy, D. Zosso. Variational Mode Decomposition. Transaction on signal processing. 2014, 62, 531-544.

30. MA. Colominas, G. Schlotthauer, ME. Torres. Improved complete ensemble EMD: A suitable tool for biomedical signal processing. Biomedical Signal Processing and control. 2014, 14, 19-29 